\title{
04 SUICIDE PREVENTION: CONFRONTING PUBLIC HEALTH CHALLENGES
}

doi:10.1136/injuryprev-2012-040580m.4

E Caine. University of Rochester Medical Center, USA

Suicide has been identified as a major international public health priority. This presentation will examine 'core challenges' that impede the successful development and dissemination of effective prevention initiatives.

Data from the US and other countries will be used to illustrate key discussion points. Apparent challenges must be frankly and creatively addressed if we propose to prevent suicide at national, community, or institutional levels. The presentation draws upon lessons gleaned from both effective and less successful approaches, and considers future opportunities.

Suicide is preventable! Future initiatives must integrate injury prevention and mental health perspectives, identify common risks, and create and sustain a mosaic of coordinated programmes in order to enhance community health and promote mental health, and to provide timely access to services across diverse social settings.

\section{LEADING WORKPLACE SAFETY}

doi:10.1136/injuryprev-2012-040580m.5

C Ellis. Brightwater Group, New Zealand

Leading workplace safety demands a different approach from most other business functions. Safety performance relies on minute by minute decisions by individuals in a workforce. A moment of distraction may cause an incremental reduction in productivity, but that same inattention has the potential to cause a serious workplace injury or fatality. While the individual drivers of workplace safety behaviour are complex many businesses have been successful by addressing safety culture as a whole. Workplace health and safety must be led as well as managed.

Today most leaders of large businesses recognise the engagement, productivity and cost benefits from actively leading workplace health and safety. The challenge is that while they have a desire to improve workplace health and safety, many business leaders are uncertain what their role is and where their effort will be most effective.

The Business Leaders Health and Safety Forum brings together leaders of over 100 of New Zealand's largest companies with the vision of Zero Harm Workplaces. Members acknowledge that they are in a unique position to improve workplace health and safety in their own businesses and across the wider business community. The goal is to lead a step change in health and safety performance over the next 2 years by:

- developing and growing world-class safety leadership in New Zealands business leaders

- using the individual and collective influence of business leaders to positively contribute to changes in health and safety in New Zealand

- providing opportunities for business leaders to share resources to overcome common challenges. 